\title{
Transição de Fase Difusa e Propriedades Dielétricas de Cerâmicas
}

\section{Ferroelétricas de $(\mathrm{Pb}, \mathrm{Ba}) \mathrm{Nb}_{2} \mathrm{O}_{6}$}

\author{
I.A. Santos ${ }^{a}$, V.L. Arantes ${ }^{b}$, D. Garcia $^{a}$, J.A. Eiras $^{a}$ \\ ${ }^{a}$ Grupo de Cerâmicas Ferroelétricas, Departamento de Física, \\ Universidade Federal de São Carlos, 13565-905 São Carlos - SP, Brazil \\ ${ }^{b} I P \& D$, Universidade do Vale do Paraíba \\ 12245-170 São José dos Campos - SP, Brazil
}

Received: July 27, 2000; Revised: December 29, 2001

\begin{abstract}
Tungsten bonze structured lead barium niobate $\left(\mathrm{Pb}_{1-\mathrm{x}} \mathrm{Ba}_{\mathrm{x}} \mathrm{Nb}_{2} \mathrm{O}_{6}\right)$ ferroelectric ceramics were prepared by the conventional mixed oxide method and their dielectric properties were carefully investigated. All studied samples presented elevated values of maximum dielectric constant $\left(\varepsilon_{\mathrm{m}}^{\prime}\right)$ and its corresponding temperature of maximum $\left(\mathrm{T}_{\mathrm{m}}\right)$ in comparison with perovskite structured ones. The phase transition features indicate that the PBN samples do not present a complete diffuse phase transition. Indeed, the diffuseness degree of the ferroelectric phase transition is highly dependent of the ions $\mathrm{Pb}^{2+}$ content in the sample compositions. The diffuseness of the ferroelectric phase transition is enhanced while the temperature of maximum dielectric constant is decreased with the $\mathrm{Pb}^{2+}$ content decreasing. In addition, the maximum dielectric constant shows an opposite behavior.
\end{abstract}

Keywords: diffuse phase transition, electroceramics, niobates

\section{Introdução}

Materiais ferroelétricos que possuem contorno de fases morfotrópico (CFM) têm sido objeto de intensas pesquisas nas últimas décadas ${ }^{1}$. Cerâmicas que possuem estrutura tipo tungstênio bronze (TB) e CFM apresentam excelentes propriedades dielétricas e ferroelétricas e podem ser utilizadas em diversas aplicações práticas. As cerâmicas a base de $\mathrm{Pb}_{\mathrm{x}} \mathrm{Ba}_{1-\mathrm{x}} \mathrm{Nb}_{2} \mathrm{O}_{6}(\mathrm{PBNx}$, para 0,21< $<1,00)$ inserem-se nesse contexto e um grande esforço vem sendo empregado no sentido de se avançar nas pesquisas desse material, tanto do ponto de vista de sua preparação quanto do ponto de vista de sua caracterização física ${ }^{2,3}$. O PBN possui uma estrutura do tipo TB parcialmente preenchida, apresentando efeitos piezoelétricos intensos e altas temperaturas de máxima constante dielétrica $\left(\mathrm{T}_{\mathrm{m}}\right)$, quando comparado ao $\mathrm{BaTiO}_{3}$, que é um ferroelétrico clássico ${ }^{3}$.

A célula unitária tungstênio bronze ${ }^{2}$ (plano $a-b$ ), com simetria tetragonal ( $4 / \mathrm{mmm}$ na fase paraelétrica) e fórmula química $(\mathrm{Pb}, \mathrm{Ba})_{5} \mathrm{Nb}_{10} \mathrm{O}_{30}$, está ilustrada na Fig. 1. Nessa célula unitária cinco sítios cristalinos distintos podem ser identificados. Os sítios B1 e B2 estão inseridos no interior dos octaedros de oxigênio e são ocupados somente por átomos de $\mathrm{Nb}$. Esses octaedros permanecem ligeiramente

*e-mail: pias@ iris.ufscar.br

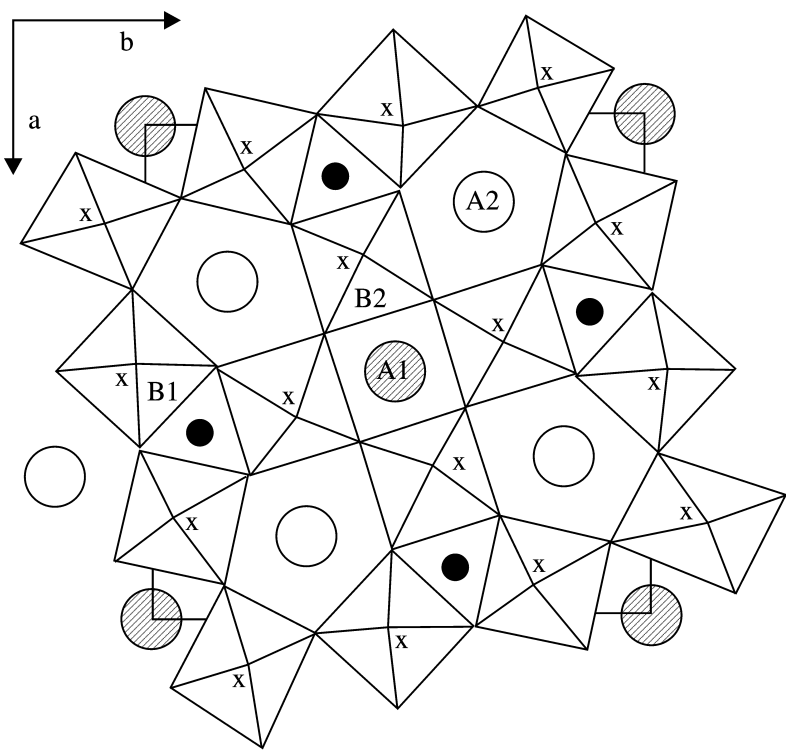

$\mathrm{x}$ : Sítio alternativo para o átomo de $\mathrm{O}$ nos Sítio c: octaedros formados com átomos de $\mathrm{Nb}$ no centro

vacante

Sítio preferencial do átomo $\mathrm{Pb}(\mathrm{z}=0,5)$

Sítio desordenado $\mathrm{Pb}$ ou $\mathrm{Ba}(\mathrm{z}=0,5)$

Figura 1. Célula unitária tungstênio bronze com simetria tetragonal (4/mmm). Os sítios B1, B2, A1, A2 e C estão destacados². 
inclinados com relação ao eixo $c$ da célula unitária, dando origem a três túneis distintos dentro da mesma, que são os sítios A1, A2 e C. No PBN o sítio C é vacante, enquanto os sítios A1 são ocupados preferencialmente por átomos de $\mathrm{Pb}$ e os sítios $\mathrm{A} 2$ (sítios desordenados) por átomos de $\mathrm{Ba}$ ou $\mathrm{Pb}$. Uma vez que somente cinco cátions de $\mathrm{Pb}^{2+} \mathrm{e} \mathrm{Ba}^{2+}$ devem ser distribuídos nos seis sítios A da célula unitária, um desses sítios permanece vacante, tornando a célula unitária parcialmente preenchida ${ }^{2}$. Composições de (x) $\mathrm{PbNb}_{2} \mathrm{O}_{6}(1-\mathrm{x}) \mathrm{BaNb}_{2} \mathrm{O}_{6}$ constituem uma solução sólida com estrutura ortorrômbica para $0,63<\mathrm{x}<1,00$ e tetragonal para $0,21<x<0,63$. Na região de composições próximas a $\mathrm{x} \sim 0,63$, o PBN apresenta um CFM, no qual a duas estruturas cristalinas podem coexistir (ver Fig. 2). Xiao e colaboradores $^{3}$, através de medidas dielétricas e microscopia eletrônica de transmissão, estudaram os efeitos relacionados aos estados de ordem-desordem dos sítios A da célula unitária TB de cerâmicas de PBN, na região do CFM, em função de diferentes condições de tratamentos térmicos. Uma região de superestrutura incomensurável foi observada. Os mesmos autores ${ }^{1}$, dando continuidade a esses estudos, observaram uma grande dispersão das temperaturas de máximo da constante dielétrica com a freqüência na região do CFM em amostras submetidas choques térmicos. Por outro lado, amostras submetidas a tratamentos térmicos, ou mesmo resfriadas lentamente, não apresentaram tais características. Esses resultados foram associados aos estados de ordem-desordem na estrutura TB das cerâmicas estudadas, ou seja, na distribuição de vacâncias entre os sítios A1 e A2. Guo e colaboradores ${ }^{5}$ estudaram os efeitos relacionados aos mecanismos de polarização em PBN mono e policristalino preparado na região do CFM. Desses estudos resultou a identificação de regiões de domínios ferroelásticos devido a superestruturas incomen-

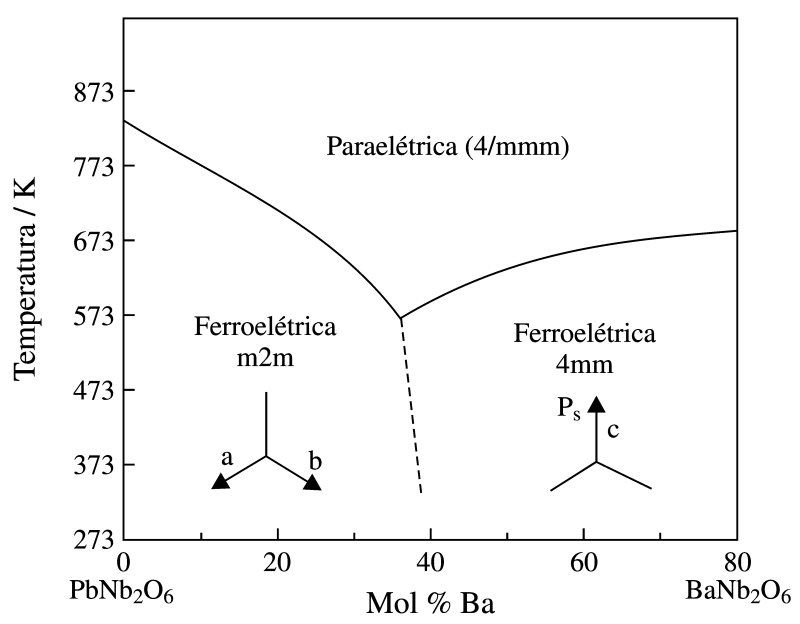

Figura 2. Diagrama de fases do sistema pseudo-binário $\mathrm{PbNb}_{2} \mathrm{O}_{6}-$ $\mathrm{BaNb}_{2} \mathrm{O}_{6}[3]$. suráveis na região de composições estudada, sendo que os mecanismos de polarização foram discutidos sob o ponto de vista termodinâmico, considerando a coexistência de fases na região do CFM. A influência das características microestruturais sobre as propriedades físicas de cerâmicas de $\mathrm{PBN}$, preparadas pelo método convencional de sinterização ou por densificação assistida por pressão via prensagem uniaxial a quente, foi investigada por Hiroshima e colaboradores $^{7}$. Esses estudos revelaram que a temperatura de máxima constante dielétrica do material é altamente dependente da distribuição de tamanho de grãos na cerâmica.

A despeito de suas excelentes propriedades físicas, aliadas a grandes potencialidades para aplicações tecnológicas, uma quantidade muito pequena de trabalhos relacionados ao estudo das características e aspectos básicos das transições de fase em materiais ferroelétricos com estrutura TB pode ser encontrada na literatura. Como relação ao PBN, esse número é ainda muito mais escasso. $\mathrm{O}$ objetivo deste trabalho é apresentar e discutir as propriedades dielétricas de cerâmicas de PBN preparadas próximas ao CFM. A resposta dielétrica a baixo campo foi estudada em função da temperatura e da freqüência e os principais aspectos da transição de fase difusa, apresentada por esse material, foram analisados lançando mão de uma descrição fenomenológica, voltada para transições de fase em materiais ferroelétricos, recentemente proposta por Santos e Eiras ${ }^{8}$.

\section{Procedimento Experimental}

As amostras utilizadas neste trabalho foram obtidas próximas ao CFM do sistema PBN através do método cerâmico convencional de mistura de precursores óxidos. As matérias-primas utilizadas foram: $\mathrm{PbO}, \mathrm{Nb}_{2} \mathrm{O}_{5}$ e $\mathrm{Ba}\left(\mathrm{NO}_{3}\right)$, todos óxidos com pureza analítica (Aldrich). Essas matérias-primas foram misturadas segundo a fórmula $\left(\mathrm{Pb}_{\mathrm{x}} \mathrm{Ba}_{1-\mathrm{x}}\right) \mathrm{Nb}_{2} \mathrm{O}_{6}$, com $\mathrm{x}=0,60,0,63$ e 0,66 . Os pós foram misturados em moinho de bolas por $24 \mathrm{~h}$, usando potes de polietileno e cilindros de zircônia estabilizada. Utilizou-se álcool isopropílico como solvente. Os pós foram calcinados a temperatura de $1473 \mathrm{~K}$ por $4 \mathrm{~h}$. A fim de se obter tamanhos de partícula adequados a densificação do material (abaixo de $1 \mu \mathrm{m}$ ), foram necessárias $60 \mathrm{~h}$ de moagem em moinho de bolas. O tamanho médio de partículas, obtido com a utilização de um sedígrafo Horiba CAPA700, foi de $(0,56 \pm 0,08) \mu \mathrm{m}$. Maiores detalhes a respeito da preparação e evolução da formação de fases no sistema PBN podem ser encontrados na referência ${ }^{9}$. Os corpos cerâmicos foram obtidos pelo método convencional de sinterização como previamente reportado ${ }^{10}$. Os pós foram inicialmente conformados na forma de discos de $15 \times 2 \mathrm{~mm}^{2}$ via prensagem uniaxial e isostática a frio. A 
sinterização das amostras foi realizada a $1523 \mathrm{~K}$ por $4 \mathrm{~h}$. Suas estequiometrias foram determinadas através de análise por microscopia eletrônica de varredura, utilizandose um microscópio Phillips FEG XL equipado com espectroscopia dispersiva de raios-X (EDX), revelando as seguintes razões $\mathrm{Pb} / \mathrm{Ba}$ : 59/41 (PBN59), 61/39 (PBN61) e 63/37 (PBN63). Essas composições encontram-se na região de estrutura tetragonal $(4 \mathrm{~mm})$ do diagrama de fases ${ }^{3}$ da solução sólida $\mathrm{PbNb}_{2} \mathrm{O}_{6}-\mathrm{BaNb}_{2} \mathrm{O}_{6}$, que está ilustrado na Fig. 2. Como podemos perceber, as amostras sofreram uma contínua perda de $\mathrm{Pb}$ em relação às composições iniciais e aquelas determinadas através da análise por EDX. As amostras sinterizadas foram polidas com SiC 1500 mesh. Para efetuarmos a análise dielétrica, eletrodos de Au foram depositados nas superfícies das amostras via sputering. Tais análises foram efetuadas com a utilização de um analisador de impedâncias HP4194A, num intervalo de temperaturas de $300 \mathrm{~K}$ a $750 \mathrm{~K}$ (com precisão de $0,1 \mathrm{~K}$ nesse intervalo de temperaturas) e num intervalo de freqüências de $1 \mathrm{kHz}$ a $1 \mathrm{MHz}$.

\section{Fundamentação Teórica}

A caracterização da dependência da constante dielétrica com a temperatura em materiais ferroelétricos tem sido extensivamente estudada, revelando-se como uma importante e indispensável ferramenta para a determinação das características básicas das transições de fase desses materiais. Desta forma, numerosos esforços vêm sendo empregados no sentido de promover essa caracterização a temperaturas superiores a temperatura de máxima constante dielétrica ${ }^{11}$, já que nessa região de temperaturas e especificamente no intervalo de rádio freqüências, nenhuma dispersão com a frequiência para as temperaturas de máximo da constante dielétrica é observada. A difusividade de uma transição de fase ferroelétrica pode ser determinada por variados métodos, sendo que o mais empregado, para temperaturas superiores a $T_{m}$ e que se revela em boa concordância com os resultados experimentais, é a aproximação Gaussiana dada abaixo. Essa aproximação, originalmente proposta por Kirillov e Isupov ${ }^{12}$, considera que as micro regiões polares do material não possuem a mesma composição química, isto é, existem flutuações composicionais. Sendo assim, cada região polar sofre a transição de fase numa dada temperatura dentro de um intervalo de temperaturas tomado ao redor de $\mathrm{T}_{\mathrm{m}}$. Desta forma, uma distribuição Gaussiana pode ser utilizada, na qual a interação entre as regiões polares não é considerada. A difusividade (parâmetro $\delta$ ) de uma transição de fase ferroelétrica pode ser determinada através do ajuste das curvas da constante dielétrica em função da temperatura com a seguinte expressão:

$$
\varepsilon^{\prime}=\frac{\varepsilon_{\mathrm{m}}^{\prime}}{1+\frac{1}{2}\left(\frac{\mathrm{T}-\mathrm{T}_{\mathrm{m}}}{\delta}\right)^{2}}
$$

sendo que na equação dada acima $\varepsilon$ 'm é a máxima constante dielétrica, $\mathrm{T}_{\mathrm{m}}$ a temperatura de máxima constante dielétrica e $\delta$ o parâmetro que nos indica o grau de difusividade da transição de fase, dando informações a respeito da largura da transição. Contudo, a Eq. 1 revela-se altamente dependente da freqüência analisada e ainda não faz distinção entre compostos e soluções sólidas ${ }^{13}$.

Recentemente, uma nova aproximação, que se revela ainda mais abrangente que a Eq. 1 com relação ao estudo de transições de fase em ferroelétricos, foi proposta por Santos e Eiras ${ }^{8}$. Essa aproximação tem como característica básica o fato de considerar, do ponto de vista mesoscópico, a presença de regiões ferroelétricas (regiões polares) interagentes a temperaturas superiores a $T_{m}$ (ou $T_{c}$ no caso de transições de fase de primeira ou segunda ordem e que podem ser descritas pela teoria de Landau-Devonshire). Levando-se em consideração este importante aspecto de materiais com transição de fase difusa e/ou comportamento relaxor $^{1}$, a seguinte expressão pode ser utilizada para a parametrização da transição de fase de materiais ferroelétricos:

$$
\varepsilon^{\prime}=\frac{\varepsilon_{\mathrm{m}}^{\prime}}{1+\left(\frac{\mathrm{T}-\mathrm{T}_{\mathrm{m}}}{\Delta}\right)^{\xi}}
$$

sendo que nessa equação $\xi=1$ corresponde ao comportamento tipo Curie-Weiss "normal" para a transição de fase e $\xi=2$ corresponde a uma transição de fase "completamente" difusa (ver discussão abaixo). Como podemos observar, no limite $\xi \rightarrow 2$, a Eq. 2 torna-se praticamente similar à Eq. 1. Todavia, a Eq. 2 possui a grande vantagem de apresentar resultados independentes da freqüência analisada, figurando ainda como a única aproximação existente na literatura que é capaz de ajustar as curvas de $\varepsilon^{\prime}$ vs $\mathrm{T}$ na região de dispersão dielétrica, ou seja, em torno de $\mathrm{T}_{\mathrm{m}}$. A interpretação física dos parâmetros $\Delta$ e $\xi$, obtidos através da Eq. 2, pode ser descrita sucintamente na forma:

1 - O parâmetro $\Delta$ nos fornece informações a respeito da difusividade da transição de fase, já que ele está diretamente ligado a largura da curva de $\varepsilon^{\prime}$ vs T;

2 - O parâmetro $\xi$ nos fornece informações a respeito do caráter da transição de fase, ou seja, se ela é "completamente" difusa, "parcialmente" difusa ou se assume um 
caráter ao qual designamos de "normal", no qual a lei de Curie-Weiss para a dependência da permissividade dielétrica com o inverso da temperatura é obedecida.

A interpretação física relacionada com os parâmetros $\Delta$ e $\xi$, nas distintas situações na qual a Eq. 2 se aplica, pode ser encontrada em detalhes na referência ${ }^{8}$. A seguir apresentamos uma sucinta descrição da mesma.

Quando um material que apresente comportamento tipo Curie-Weiss "normal" é resfriado da região de temperaturas da fase paraelétrica para a região de temperaturas da fase ferroelétrica, as regiões polares no material serão formadas num intervalo muito estreito de temperaturas (situado próximo a $\mathrm{T}_{\mathrm{m}}$, que nesse caso específico será a temperatura de Curie-Weiss, $\mathrm{T}_{0}$ ). Essa dinâmica de formação de regiões polares torna a curva $\varepsilon$ ' $v s$ T muito estreita e, conseqüentemente, o valor do parâmetro $\Delta$ tende a diminuir quando empregamos a Eq. 2 na parametrização das curvas de $\varepsilon^{\prime} v s$ T nesse tipo de material. Do ponto de vista da dinâmica de interação entre essas regiões polares, podemos dizer que ela surge de uma forma muito intensa e essa intensidade aumenta num intervalo de tempo muito curto quando o material se aproxima da temperatura de transição, fazendo com que $\xi$ assuma valores próximos a unidade, refletindo assim o comportamento tipo Curie-Weiss "normal" para a dependência da permissividade dielétrica com o inverso da temperatura.

Por outro lado, quando um material que apresente transição de fase "completamente" difusa é resfriado da região paraelétrica para a região ferroelétrica, as regiões polares começam a ser formadas a temperaturas muito superiores a $\mathrm{T}_{\mathrm{m}}{ }^{14}$. Todavia, a interação entre essas regiões dá-se de uma maneira muito tênue, por vezes tornando-se completamente frustrada. Sendo assim, a curva $\varepsilon$ ' $v s$ T torna-se muito ampla e, conseqüentemente, $\Delta$ assumirá um valor elevado. Como a interação entre as regiões polares é muito fraca ou mesmo inexistente, $\xi$ tende a valores próximos a 2, o que caracteriza a transição de fase completamente difusa.

Vamos analisar a situação na qual o material não apresente transição de fase completamente difusa. Quando um material ferroelétrico com essa característica é resfriado da região paraelétrica para a região ferroelétrica, a quantidade de micro regiões polares, que começam a ser formadas com o resfriamento, começa a aumentar gradativamente e, conseqüentemente, a interação entre essas regiões começa a intensificar-se com o decréscimo da temperatura. Dessa forma, podemos esperar que o valor do parâmetro que caracteriza a transição de fase quanto ao seu caráter, isto é, $\xi$, situe-se entre seus valores limites, ou seja, 1 e 2 . Esse cenário parece ser o mais condizente com aquele realmente observado e conjeturado para materiais ferroelétricos que apresentam transição de fase difusa (como a maioria dos ferroelétricos relaxores, por exemplo). Como veremos a seguir, o PBN figura dentre os materiais ferroelétricos com tais características.

\section{Resultados e Discussão}

Na Fig. 3 estão ilustradas as curvas de constante dielétrica, medidas em função da temperatura e da freqüência, para as amostras PBN59, PBN61 e PBN63. Os valores de $\mathrm{T}_{\mathrm{m}}$ observados nessas amostras diminuem com o aumento da quantidade de íons $\mathrm{Pb}^{2+}$ em suas composições. Observando ainda as respectivas curvas, notamos um efeito inverso nos valores de máxima constante dielétrica $\left(\varepsilon^{\prime}\right.$ m) com relação à quantidade de $\mathrm{Pb}^{2+}$, isto é, $\varepsilon^{\prime}$ m é maior nas amostras com maiores quantidades de chumbo. Esses resultados estão de acordo com os previamente reportados e são explicados levando-se em consideração o comportamento tipo ordem-desordem nos sítios A da célula unitária TB e a quantidade de $\mathrm{Pb}^{2+}$ na composição do material ${ }^{6}$. À medida que a quantidade de $\mathrm{Pb}^{2+}$ aumenta, a desordem no sítio A2 também é aumentada, tendo em vista o fato de que esse sítio pode ser ocupado tanto por íons de

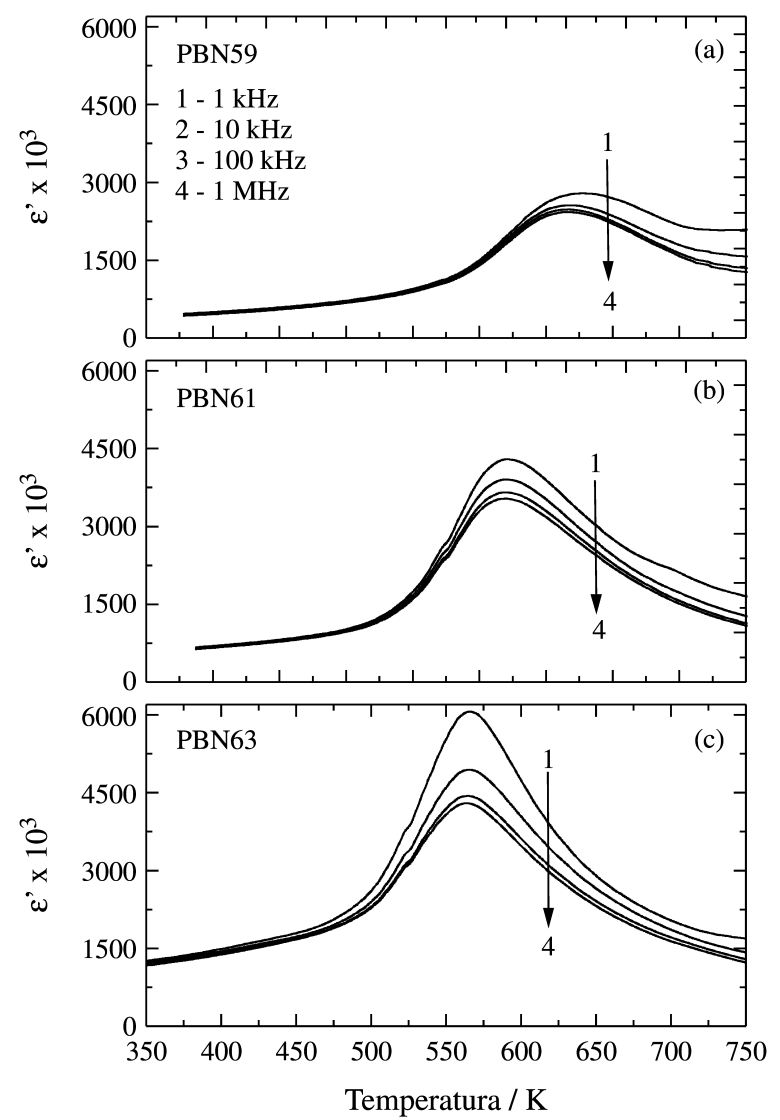

Figura 3. Constante dielétrica em função da temperatura e frequiência

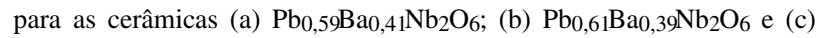
$\mathrm{Pb}_{0,63} \mathrm{Ba}_{0,37} \mathrm{Nb}_{2} \mathrm{O}_{6}$. 
bário quanto por íons de chumbo. $\mathrm{O}$ aumento da desordem na ocupação dos sítios A2 da célula unitária também promove uma maior distorção da mesma, e conseqüentemente uma polarização mais intensa (mudanças de polarizibilidade no sítio A2). Sendo assim, na fase tetragonal (simetria 4mm, Fig. 2 - lado direito) e com o aumento da quantidade de $\mathrm{Pb}^{2+}$ (aproximando as composições do CFM), as amostras de PBN apresentariam menores temperaturas de máximo e valores mais elevados de máxima constante dielétrica, corroborando com outros resultados da literatura ${ }^{4}$. Por outro lado, com o aumento contínuo da quantidade de $\mathrm{Pb}^{2+}$, e com o distanciamento do CFM (na fase ortorrômbica, simetria m2m - Fig. 2 - lado esquerdo), o efeito se inverte, ou seja, os valores das máximas constantes dielétricas tendem a diminuir enquanto suas respectivas temperaturas de máximo aumentam.

Nossa interpretação para esse caráter de ocupação dos sítios $\mathrm{A} 2$ pelos íons $\mathrm{Pb}^{2+}$ é corroborada pela dependência típica das temperaturas de máxima constante dielétrica com a frequiência. Como podemos observar (Fig. 3) a dependência de $\mathrm{T}_{\mathrm{m}}$ com a freqüência, em todas as amostras, revela uma dispersão dielétrica muito tênue, não ultrapassando $2 \mathrm{~K}$ para freqüências variando de $1 \mathrm{kHz}$ a $1 \mathrm{MHz}$. Esse pequeno intervalo de dispersão dielétrica com a freqüência nos indica que essas amostras possuem um melhor ordenamento do ponto de vista de ocupação dos sítios A da célula unitária TB em relação aos resultados anteriormente reportados. Além disso, ela também insinua a possibilidade de um aumento do tamanho dos sítios A com o resfriamento lento efetuado após a sinterização do material, corroborando com os resultados amplamente divulgados na literatura $^{15,16}$. Contudo, a dependência com a frequiência para as amplitudes da constante dielétrica pode estar também associada a contribuições de paredes de domínios ou ainda a possíveis efeitos de ordem-desordem nos sítios A da célula unitária, todavia, nossas análises não nos permitem fazer maiores indagações a esse respeito.

Com o objetivo de analisar as principais características da transição de fase do PBN, efetuamos o ajuste das curvas de constante dielétrica em função da temperatura (determinadas a $10 \mathrm{kHz}$ ) utilizando as Eqs. 1 e 2. Os ajustes foram realizados tomando somente as regiões de alta temperatura, isto é, a temperaturas superiores às temperaturas de máxima constante dielétrica de cada amostra. As curvas geradas através dos parâmetros obtidos com as Eqs. 1 e 2 estão ilustradas na Fig. 4. Nessa figura verificamos que as curvas geradas através dos parâmetros obtidos com a utilização da Eq. 2 são mais condizentes com os resultados experimentais do que as curvas geradas através dos parâmetros da Eq. 1. Os parâmetros obtidos para as amostras de PBN, tanto experimentalmente, quanto através das Eqs. 1 e 2, estão listados na Tabela 1. Como podemos observar, os

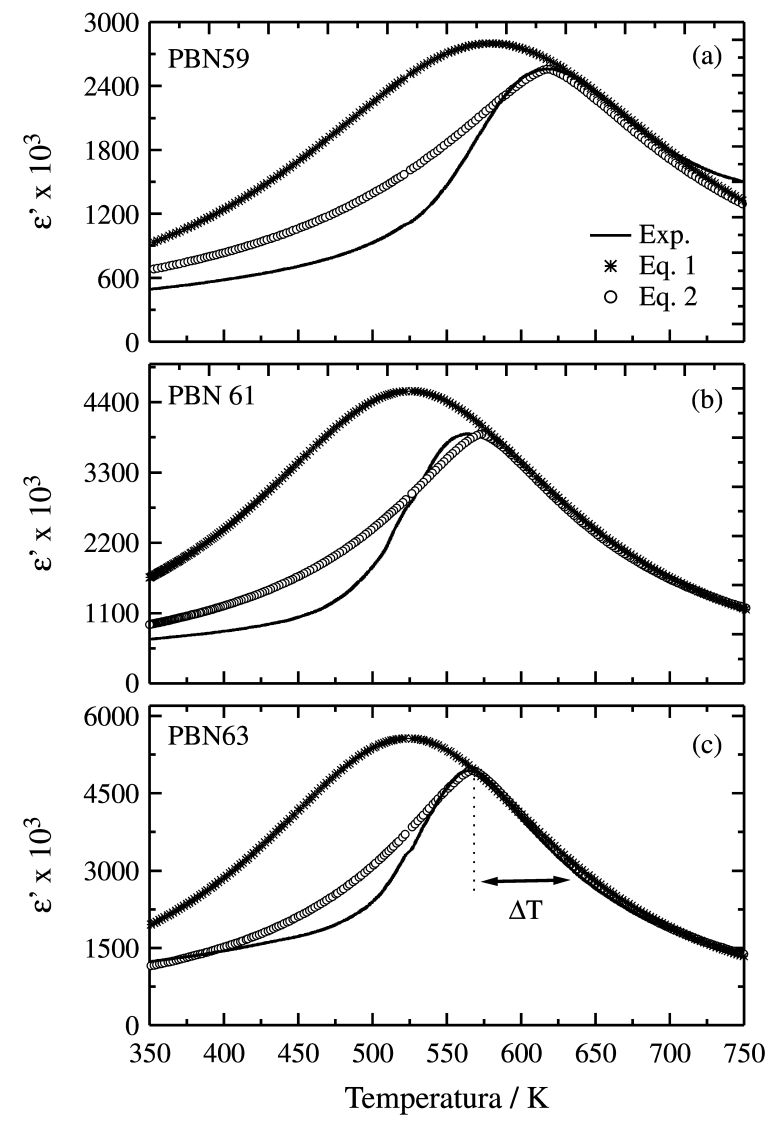

Figura 4. Constante dielétrica em função da temperatura à frequiência

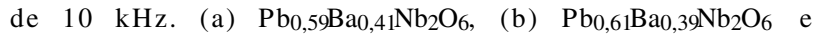
(c) $\mathrm{Pb}_{0,63} \mathrm{Ba}_{0,37} \mathrm{Nb}_{2} \mathrm{O}_{6}$. Nessas ilustrações estão apresentadas as curvas de E'vs T geradas através dos parâmetros de ajuste das Eqs. 1 e 2.

Tabela 1. Parâmetros experimentais e de ajuste (utilizando as Eqs. 1 e 2) obtidos das curvas de $\varepsilon$ ' $v s$ T das amostras de PBN.

\begin{tabular}{lccc}
\hline Experimental & $\mathcal{E}^{\prime} \mathrm{m}$ & $\mathrm{T}_{\mathrm{m}}(\mathrm{K})$ & $\Delta \mathrm{T}(\mathrm{K})$ \\
\hline PBN59 & 2559 & 617,1 & 109,2 \\
PBN61 & 3905 & 564,8 & 90,5 \\
PBN63 & 4943 & 564,3 & 70,7 \\
\hline
\end{tabular}

\begin{tabular}{lcccc}
\hline Eq. 1 & $\mathcal{E}^{\prime} \mathrm{m}$ & $\mathrm{T}_{\mathrm{m}}(\mathrm{K})$ & $\delta(\mathrm{K})$ & $\Gamma_{\delta}(\mathrm{K})$ \\
\hline PBN59 & 4572 & 579,6 & 113,4 & 133,5 \\
PBN61 & 4572 & 524,9 & 93,3 & 109,8 \\
PBN63 & 5565 & 523,7 & 89,8 & 105,7 \\
\hline
\end{tabular}

\begin{tabular}{lccccc}
\hline Eq. 2 & $\varepsilon_{\mathrm{m}}^{\prime}$ & $\mathrm{T}_{\mathrm{m}}(\mathrm{K})$ & $\Delta(\mathrm{K})$ & $\Gamma \xi(\mathrm{K})$ & $\xi$ \\
\hline PBN59 & 2558 & 618,3 & 128,9 & 103,6 & 1,66 \\
PBN61 & 3902 & 566,2 & 89,7 & 89,7 & 1,60 \\
PBN63 & 4942 & 565,2 & 73,9 & 73,9 & 1,55 \\
\hline
\end{tabular}

parâmetros físicos $\left(\varepsilon_{\mathrm{m}}^{\prime}\right.$ e $\left.\mathrm{T}_{\mathrm{m}}\right)$ obtidos através da Eq. 2 também apresentam uma melhor concordância com os 
resultados experimentais do que aqueles obtidos com a Eq. 1. Observa-se também que a qualidade do ajuste realizado com a Eq. 2 é incontestavelmente mais elevada do que o ajuste conseguido com a Eq. 1. Como anteriormente afirmado, a Eq. 1 considera transições de fase completamente difusas, e desta forma, não pode ser aplicada na análise das características da transição de fase do PBN, conforme demonstraremos a seguir.

Outro parâmetro que pode ser utilizado para comparar a eficácia da análise da transição de fase do material estudado utilizando as Eqs. 1 e 2 é a determinação da meia largura a meia altura da curva de constante dielétrica e sua comparação com a meia largura a meia altura das curvas geradas com a utilização dos parâmetros de ajuste conseguidos através dessas equações. Como a análise é feita somente nas regiões de temperaturas superiores a $\mathrm{T}_{\mathrm{m}}$ (que é a região de interesse), tomamos por regra medir $\Delta \mathrm{T}$ como indicado na Fig. 4(c). Sendo assim, podemos comparar os valores de $\Gamma_{\exp }=\Delta \mathrm{T}$ com os valores das curvas de ajuste, que são dados por: $\Gamma_{\delta}=\delta(2 \ln 2)^{1 / 2}$, para a Eq. 1 , e $\Gamma_{\xi}=\Delta(\ln 2)^{1 / \xi}$, para a Eq. 2, respectivamente ${ }^{8}$. Os valores de $\Gamma_{\exp }, \Gamma_{\delta}$ e $\Gamma_{\xi}$ também estão listados na Tabela 1. Comparando esses valores, observamos uma clara semelhança entre os parâmetros $\Gamma_{\exp }$ e $\Gamma_{\xi}$, corroborando nossa afirmação de que a Eq. 2 é a mais apropriada para se estudar o caráter da transição de fase de materiais ferroelétricos com transição de fase parcialmente difusa.

Analisando os resultados apresentados na Tabela 1 observamos que na amostra PBN63 o processo de formação de regiões polares, assim como a interação entre essas regiões é mais intensa do que o apresentado pelas amostras PBN59 e PBN61. Esse fato é confirmado pelos valores dos parâmetros $\Delta \mathrm{e} \xi$ apresentados por essa amostra, que são inferiores aos valores apresentados pelas demais amostras. À medida que a quantidade de chumbo diminui, o parâmetro $\xi$ aumenta, assumindo os valores 1,55, 1,60 e 1,66 para as amostras PBN63, PBN61 e PBN59, respectivamente. Contudo, podemos observar que os valores se mantêm em um nível intermediário, isto é, entre 1 e 2. Esse resultado corrobora com nossa afirmação a respeito do caráter da transição de fase desse material, ou seja, o PBN possui uma transição de fase parcialmente difusa.

Ainda no intuito de averiguar a validade da utilização da Eq. 2 no estudo das características das transições de fase em materiais ferroelétricos, traçamos os gráficos da constante dielétrica normalizada ( $\left.\varepsilon^{\prime} / \varepsilon_{\mathrm{m}}^{\prime}\right)$ em função da temperatura normalizada $\left(\mathrm{T} / \mathrm{T}_{\mathrm{m}}\right)$ para todas as amostras estudadas. Esses resultados estão ilustrados na Fig. 5. Observamos claramente nessa figura que a amostra PBN59 apresenta uma curva mais "larga" do que as demais amostras. De fato, a amostra PBN63 apresenta a curva mais "estreita". Sendo

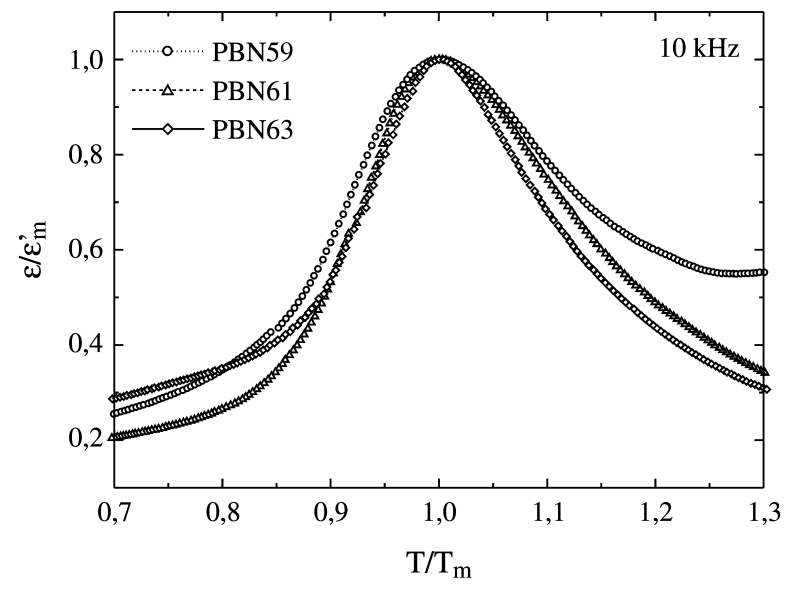

Figura 5. Constante dielétrica normalizada ( $\left.\varepsilon^{\prime} / \varepsilon^{\prime} \mathrm{m}\right)$ em função da tem-

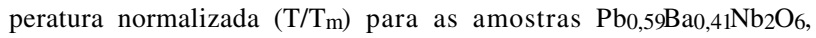

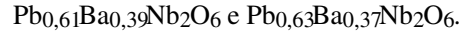

assim, podemos inferir que a difusividade da transição de fase de amostras de PBN, preparadas na região do CFM desse sistema, é maior para amostras com menor quantidade de $\mathrm{Pb}$ em sua composição, como havíamos afirmado anteriormente através da análise das curvas de constante dielétrica em função da temperatura com a utilização da Eq. 2.

Esse comportamento para a transição de fase difusa do PBN pode ser mais bem entendido considerando aspectos relacionados tanto com sua estrutura $\mathrm{TB}$, quanto com a ocupação dos interstícios (sítios A) das células unitárias pelos átomos de $\mathrm{Pb}$. De fato, esses efeitos foram considerados em um trabalho recente, concernente às transições de fase do sistema $\mathrm{PBN}^{17}$, que aprofunda a análise do comportamento dielétrico desse material considerando aspectos relacionados ao seu nível mesoscópico. Tomando como base às idéias propostas por Glinchuk e Stephanovich ${ }^{18}$, nas quais defeitos na rede cristalina (tais como impurezas, centros de dilatação, etc) de dielétricos desordenados promovem o surgimento de intensas fontes de campos elétricos aleatórios oscilantes em suas estruturas, podemos considerar que no PBN, com o acréscimo da quantidade de íons $\mathrm{Pb}^{2+}$ rumo ao CFM (ainda na fase tetragonal), os sítios $\mathrm{A} 2$ das células unitárias TB passam também a ser ocupados por esses íons. Podemos admitir ainda que através dessa dinâmica de ocupação dos sítios $\mathrm{A} 2$, os íons $\mathrm{Pb}^{2+}$ passam a agir como centros de dilatação na estrutura cristalina do PBN. Esses centros de dilatação, por sua vez, agem intensamente no sentido de frustrar as interações de longo alcance entre as regiões polares do material via seus campos elétricos aleatórios oscilantes ${ }^{19}$. Além disso, eles também agem no sentido de diminuir a temperaturas de máximo da constante dielétrica, como podemos observar no diagrama de fases da Fig. 2 (lado direito). Contudo, com o contínuo acréscimo de $\mathrm{Pb}$, e entrando na região de simetria ortorrôm- 
bica (lado esquerdo do diagrama), os íons $\mathrm{Pb}^{2+}$ perdem esse caráter, fazendo com que um efeito inverso seja observado com relação ao comportamento das temperaturas de máxima constante dielétrica.

Esses centros de dilatação também exercem forte influência tanto na difusividade da transição de fase, via frustração da interação entre as regiões polares, quanto nos valores de máximo da constante dielétrica. Usualmente, eles agem no sentido de aumentar a difusividade da transição. Contudo, no PBN, um efeito oposto é observado (parâmetro $\Delta$ da Tabela 1 e Fig. 5), ou seja, na região do CFM a transição de fase ferroelétrica é menos difusa. $\mathrm{Na}$ verdade, como apontado por Santos e colaboradores ${ }^{17}$, o efeito promovido pelos centros de dilatação contrasta com aquele apresentado pelo caráter par de elétrons livres (lone pair caracter) dos íons $\mathrm{Pb}^{2+}$ na estrutura $\mathrm{TB}^{6}$. Esse caráter especial faz com aconteça uma forte hibridização entre os orbitais s e p dos átomos de oxigênio e chumbo, respectivamente, que por sua vez contribui para uma forte distorção da célula unitária na região de coexistência de fases, ou seja, no CFM. Essas distorções são altamente favorecidas pela diminuição da energia eletrocristalina nessa região específica do diagrama de fases do sistema ${ }^{6}$. Sendo assim, com o aumento da distorção das células unitárias, suas polarizações tendem a se alterar grandemente (mudanças de polarizibilidade nas vizinhanças dos sítios A2). Essas alterações de amplitude dos vetores polarização promovem uma maior interação entre as regiões polares do PBN na região do CFM, fazendo com que a difusividade da transição de fase diminua e os valores de máximo das constantes dielétricas aumentem nessa região. De fato, esse efeito é preponderante sobre àquele causado pelo caráter de centro de dilatação dos íons $\mathrm{Pb}^{2+}$ no CFM. Contudo, os centros de dilatação ainda contribuem fortemente para uma gradual diminuição das temperaturas de máxima constante dielétrica.

\section{Conclusões}

Cerâmicas de niobato de chumbo e bário (PBN) foram preparadas através do método cerâmico convencional de mistura de óxidos e suas propriedades dielétricas foram cuidadosamente investigadas em função da temperatura e freqüência. As amostras apresentaram elevados valores de máxima constante dielétrica $\left(\varepsilon^{\prime}{ }_{m}\right)$ e de sua correspondente temperatura de máximo $\left(\mathrm{T}_{\mathrm{m}}\right)$, assim como uma tênue dispersão das temperaturas de máximo com a frequiência. $\mathrm{O}$ caráter da transição de fase do PBN tetragonal foi cuidadosamente analisado utilizando-se uma aproximação fenomenológica que nos permite ajustar as curvas de constante dielétrica em função da temperatura, independen- temente da frequiência analisada. Os ajustes efetuados nos permitiram obter parâmetros que nos fornecem indicativos a respeito do caráter da transição de fase do material. Os resultados indicaram que o PBN não possui uma transição de fase completamente difusa, e que o grau de difusividade dessa transição está associado à quantidade de íons $\mathrm{Pb}^{2+}$ na composição do PBN. Observamos que as amostras que possuem uma menor quantidade de chumbo na composição apresentam uma maior difusividade da transição de fase (valores mais elevados de $\Delta \mathrm{e} \xi$ ), e mais elevadas temperaturas de máxima constante dielétrica. Com relação à máxima constante dielétrica, um efeito oposto é observado, isto é, ela aumenta à medida que a quantidade de chumbo é aumentada, ou seja, à medida que a composição se aproxima do contorno de fases morfotrópico.

\section{Agradecimentos}

Os autores expressam seu cordial agradecimento às agências CAPES e FAPESP (contratos n. 01/06045-9 e 00/09722-9) pelo apoio financeiro. Agradecemos também o suporte técnico fornecido por F.J. Picon (GCFerrUFSCar).

\section{Referências}

1. Cross, L.E. Ferroelectrics, v. 26, p. 241, 1987.

2. Framcombe, M.H. Acta Cryst., v. 13, p. 131, 1960.

3. Randall, C.A.; Guo, R.; Bhalla, A.S.; Cross, L.E. J. Mat. Res., v. 6, p. $1720,1991$.

4. Xiao, X.; Xu, Y.; Zeng, Z.; Gui, Z.; Li, L.; Zhang, X.J. Mat. Res., v. 11, p. 650, 1996.

5. Xiao, X;. Xu, Y.; Zeng, Z.; Gui, Z.; Li, L.; Zhang, X.J. Mat. Res., v. 11, p. 2302, 1996.

6. Guo, R.; Bhalla, A.S.; Randall, C.A.; Chang, Z.P.; Cross, L.E. J. Appl. Phys., v. 67, p. 1453, 1990; v. 67, p. 6405, 1990.

7. Hiroshima, T.; Tanaka, K.; Kimura, T.J. Am. Ceram. Soc., v. 79, p. 3235, 1996.

8. Santos, I.A.; Eiras, J.A. J. Phys. Cond. Matt., v. 13, p. 11733, 2001.

9. do Carmo, C.V.; de Paula, R.N.; Póvoa, J.M.; Garcia, D.; Eiras, J.A. J. Eur. Ceram. Soc., v. 19, p. 1057, 1999.

10. Arantes, V.L.; de Paula, R.N.; Santos, I.A.; Garcia, D.; Eiras, J.A. J. Mat. Sc. Lett., v. 19, p. 1677, 2000.

11. Cheng, Z.Y.; Katiyar, S.; Yao, X.; Guo, A. Phys. Rev. B, v. 55, p. 8165, 1997.

12. Kirilov, V.V.; Isupov, V.A. Ferroelectrics, v. 5, p. 3, 1972.

13. Bokov, A.A.; Ye, Z.G. S. State Comm., v. 116, p. 105, 2000.

14. Viehland, D.; Jang, S.J.; Cross, L.E.; Wuttig, M. Phys. Rev. B, v. 46, p. 8003, 1992.

15. Guo, R.; Evans, H.T.; Bhalla, A.S. Ferroelectrics, v. 206-207, p. 123, 1998.

16. Trubelja, M.P.; Ryba, E.; Smith, D.K. J. of Mat. Sc., v. 31, p. 1435, 1996.

17. Santos I.A.; Arantes, V.L.; Garcia, D.; Eiras, J.A. J. Appl. Phys., submetido para publicação.

18. Glinchik, M.D.; Stephanovich, V.A. J. Phys. Cond. Matt., v. 6, p. 6317, 1994.

19. Vugmeister, B.E.; Glinchuk, M.D. Rev. Mod. Phys. v. 82, p. 993, 1990.

FAPESP helped in meeting the publication costs of this article 